\title{
Variations in LOS and its main determinants overtime at an academic spinal care center from 2006-2019
}

\author{
Dandurand Charlotte ${ }^{1} \mathbb{D} \cdot$ N. Hindi Mathew ${ }^{1} \cdot$ Ailon Tamir $^{1} \cdot$ Boyd Michael $^{1} \cdot$ Charest-Morin Raphaële $^{1} \cdot$ Dea Nicolas $^{1}$. \\ Dvorak Marcel $^{1} \cdot$ Fisher Charles $^{1} \cdot$ K. Kwon Brian ${ }^{1} \cdot$ Paquette Scott $^{1} \cdot$ Street John ${ }^{1}$
}

Received: 31 May 2021 / Revised: 16 November 2021 / Accepted: 3 December 2021 / Published online: 11 January 2022

(c) The Author(s), under exclusive licence to Springer-Verlag GmbH Germany, part of Springer Nature 2021

\begin{abstract}
Objectives Efforts to safely reduce hospital LOS while maintaining quality outcomes and patient satisfaction are paramount. The primary goal of this study was to assess trends in LOS at a high-volume quaternary care spine center. Secondary goals were to assess trends in factors most associated with prolonged LOS.

Methods This is a prospective study of all consecutive patients admitted from January 2006 to December 2019. Data included demographics, diagnostic category (degenerative, oncology, deformity, trauma, other), LOS (mean, median, interquartile range, standard deviation, defined as days from admission to discharge), and in-hospital adverse events.

Results A total of 13,493 patients were included. Overall LOS has not changed over time with an overall median of 6.3 days $(p=0.451)$. Median LOS significantly increased for patients treated for degenerative pathology from 2.2 days in 2006 to 3.2 days in $2019(p=0.019)$. LOS has not changed for patients treated for deformity (overall median 6.8 days, $p=0.411)$, oncology (overall median 11.0 days, $p=0.051$ ), or trauma (overall median 11.8 days, $p=0.582$ ). Emergency admissions increased 3.2\%/year for degenerative pathologies $(p=<0.001)$. Mean age has increased from 48.4 years in 2006 to 58.1 years in $2019(p=<0.001)$. This trend was observed in the deformity, degenerative and trauma group, not for patients treated for oncological disease. More adverse events were significantly associated with increasing age.

Conclusion This is the first North American study to comprehensively analyze trends in LOS for spinal surgery overtime in an academic center. Overall, LOS has not changed from 2006-2019. Various factors that influence LOS appear to have balanced each other. It may also be explained by the changing epidemiology of both elective and emergency surgeries. These findings provide opportunities for intervention and improvement, targeted at the geriatric population, to reduce length of hospitalization.
\end{abstract}

Keywords Length of stay $\cdot$ Trends $\cdot$ Spine surgery $\cdot$ Age $\cdot$ Adverse events

\section{Introduction}

Shifting age demographics in society has increased the demand for spinal surgery in the elderly [1]. Notably, an increasing proportion of spinal cord injuries occur in the elderly population with falls an increasingly common cause

Dandurand Charlotte

charlotte.dandurand@alumni.ubc.ca

Street John

john.street@vch.ca

1 Combined Neurosurgical and Orthopedic Spine Program, Blusson Spinal Cord Center, University of British Columbia, 6th Floor, 818 West 10th Avenue, Vancouver, BC V5Z 1M9, Canada
$[2,3]$. Recent advances in surgical techniques, anesthesia, and intraoperative navigation systems have expanded the indications for and the number of surgeries performed [4]. With these advances, spinal surgery is a major source of health care costs making length of stay (LOS) reduction an attractive target for ensuring access to limited inpatient beds and cost reduction per treatment event [5]. Increased LOS within a specific diagnostic category may be used as a quality of care indicator given that it is correlated with postoperative complications and morbidity [6,7]. Also, prolonged length of stay has been cited has an independent risk factor for unplanned readmissions [8]. Healthcare institutions use LOS as an indicator of case complexity and hospital performance. 
In the last 15 years, many efforts have been made to decrease LOS while ensuring high-quality care for patients via clinical care pathways [9]. Many studies have reported on factors associated with increased LOS $[1,6-8,10]$. Initiatives have been made to target modifiable risk factors preoperatively, such as pulmonary evaluation and optimization or nasal decolonization. Postoperative management has been targeted to non-modifiable risk factors, such as personalized physiotherapy or early involvement of hospitalists for ongoing medical issues. Standardized protocols have been shown to reduce complications such as the incidence of thromboembolism [11-13]. Sethi et al. [14, 15] demonstrated that a protocolized dual-attending surgeon approach and live multidisciplinary preoperative screening conference significantly reduced perioperative complication rates. Enhanced recovery after surgery with an evidence-based multidisciplinary approach to perioperative management after major surgery may reduce complications and length of stay [16, 17]. Advances such as cone beam CT-based navigation and intra-wound antibiotics aimed at reducing complications [18, 19]. Presurgical physiotherapy, so-called «prehabilitation», has not yet been shown to impact postoperative outcomes $[20,21]$. Despite these numerous technological and organizational advances among others, the reductions in LOS have been inconsistently reported [9].

Although many studies have reported LOS, very little information is available about overall temporal trends. Trends in LOS for all spine surgery have previously been examined in a multicenter study in Japan over a 10-year period [4]. The present study is the first North American study to examine trends in LOS in spinal surgery. The goal of this study is to evaluate trends in LOS at a high-volume quaternary care spine center over a 14-year period. Secondary goals were to identify trends in factors strongly associated with prolonged LOS.

\section{Methods}

\section{Data source and study population}

This study was a retrospective analysis of a prospectively maintained database of consecutive adult patients who underwent spine surgery at a quaternary high-volume spinal care center from 2006 to 2019 . The study center serves a population of 5.1 million people [22]. As Covid-19 affected nearly all aspect of hospital operations in 2020, we removed 2020 data.

\section{Variables}

Diagnostic categories included are degenerative, oncology, deformity, trauma, and other. Deformity was defined if it involved a measurable coronal or sagittal deformity and/or if it involved fusion of more than five consecutive spinal levels or more than three levels of interbody fusion. Oncology was defined as primary malignant and benign spinal tumor, intradural tumor, metastatic tumor, and multiple myeloma. Admissions were classified as emergent or elective. LOS was defined by the number of calendar days from hospital admission to hospital discharge. Mean, median, standard deviation, and interquartile range for LOS were calculated yearly overall and per diagnosis. Patients were discharged if the wound was healing well and an evaluation by the multidisciplinary team deemed the patient safe to go home. Patients with continued medical or rehabilitation needs were transferred to their local hospital or to a dedicated rehabilitation center.

\section{Primary and secondary endpoints}

The main endpoint examined was trends in LOS in all spine surgery over a 14 -year period. The secondary endpoint was to examine the trends in the factors with the strongest association with prolonged LOS in the study center database overall and in each diagnostic category.

\section{Statistical analysis}

Descriptive statistics were used for all the analysis variables, overall and by diagnosis. Length of stay required a natural log transformation to normalize the PRESS residuals in linear regression models. Multiple linear regression models were created with $\log$ LOS versus the following predictor variables: year, age, adverse events, diagnosis, emergent versus elective admission. Models were fit in overall data and by diagnostic group. Regression coefficients represent the additive effects of a one-unit increase in each predictor variable on the natural log of LOS in days. Exponentiated regression coefficients represent the multiplicative effect on LOS due to a one-unit increase in the predictor variable. All statistical analyses were performed using SAS v9.4 (SAS Institute, Cary, North Carolina).

\section{Results}

A total of 13,493 patients were included. Overall, median LOS was 6.3 days with interquartile range (IQR) 2.5-15.1. Overall mean LOS was 15.8 days with standard deviation (SD) 34.0. During the study period, $34.0 \%$ of patients were treated for degenerative pathology, $24.5 \%$ were treated for trauma, $14.6 \%$ were treated for deformity and $10.4 \%$ were treated for oncology (Table 1). The proportion of patients treated for oncological pathologies has increased on average $2.6 \% /$ year $(p=<0.001)$ (Fig. 1). The proportions of patients 
Table 1 Length of stay (days) for each diagnostic category

\begin{tabular}{llrrrr}
\hline Diagnosis & Total patients (N, \%) & Mean & Median & SD & IQR \\
\hline Trauma & $3168(24.5)$ & 23.71 & 11.84 & 38.55 & 23.05 \\
Oncology & $1347(10.4)$ & 18.59 & 11.00 & 26.28 & 16.53 \\
Deformity & $1890(14.6)$ & 10.19 & 6.81 & 12.44 & 6.91 \\
Degenerative & $4397(34.0)$ & 6.41 & 3.14 & 25.90 & 5.16 \\
\hline
\end{tabular}

treated for degenerative pathology have increased on average $1.3 \% /$ year $(p=0.006)$. The proportion of patients treated for trauma has decreased on average $1.9 \% /$ year $(p=<0.001)$. The proportion of patients treated for deformity has decreased on average $2.2 \% /$ year $(p=<0.001)$. The factors with the strongest association with prolonged LOS were age (OR1.011, $p<0.001$ ), emergency admissions (OR1.615, $p<0.001$ ), and adverse events (OR2.613, $p<0.001$ ).

Emergency and elective admission were almost equal $(50.3 \%$ vs $49.7 \%)$. Emergency admissions have increased on average $2.2 \%$ /year during the study period ( $p=<0.001$ ). Within the degenerative group, emergency admissions have increased on average 3.2\%/year $(p=<0.001)$. Overall, $29.7 \%$ of all admissions for degenerative pathologies were emergencies.

Overall mean and median LOS have not significantly changed from 2006 to 2019 ( $p=0.451$ ) (Fig. 2). The median LOS has varied from 5.4 in 2006 to 6.3 days in 2019 (Table 2). The mean LOS has varied from 19.6 days in 2006 to 14.0 days in 2019. Median LOS significantly increased for patients treated for degenerative pathology from 2.2 days in 2006 to 3.2 days in 2019 ( $p=0.019$ ) (Fig. 3). LOS has not changed for patients treated for deformity (overall median 6.8 days, $p=0.411$ ), oncology (overall median 11.0 days, $p=0.051$ ), or trauma (overall median 11.8 days, $p=0.582$ ).

The variability in LOS has decreased over the study period: specifically, the standard deviation of mean LOS decreased from 43.5 in 2006 to 25.9 in 2019 (Table 2). The variability in LOS has increased over time for the degenerative group with a standard deviation of mean LOS increasing from 6.0 to 9.0 days over the study period. The variability in LOS has not significantly changed for deformity, oncology, and trauma.

Mean age for the entire study cohort was 53.3 years (SD 17.6) with a range from 18 to 99 years old. Mean age has significantly increased from 48.4 years in 2006 to 58.1 years in $2019(p=<0.001)$. For reference, in our catchment area, the mean age of the population has increased from 40.8 to 42.7 years over the same time. In this study, the proportion of patients who were over 75 years old at the time of surgery has increased from $45.3 \%$ in 2006 to $58.2 \%$ in 2019 . The proportion of patients over 85 years old has increased from $6.6 \%$ in 2006 to $11.8 \%$ in 2019. By comparison, according to local government data, the proportion of patients over 85 years old in our catchment area has increased from 1.8 to $2.5 \%$. After adjusting for the incidence of adverse events on multivariate analysis, each additional year of age increased LOS 0.01 days $(p=<0.001)$. This means that, for the study center providing treatment to approximately 1000 patients every year, an additional 100 bed days/year is attributable to the decade increase in mean age between 2006 and 2019. Age has significantly increased over time for patients treated for deformity, degenerative pathology, and trauma. Age has

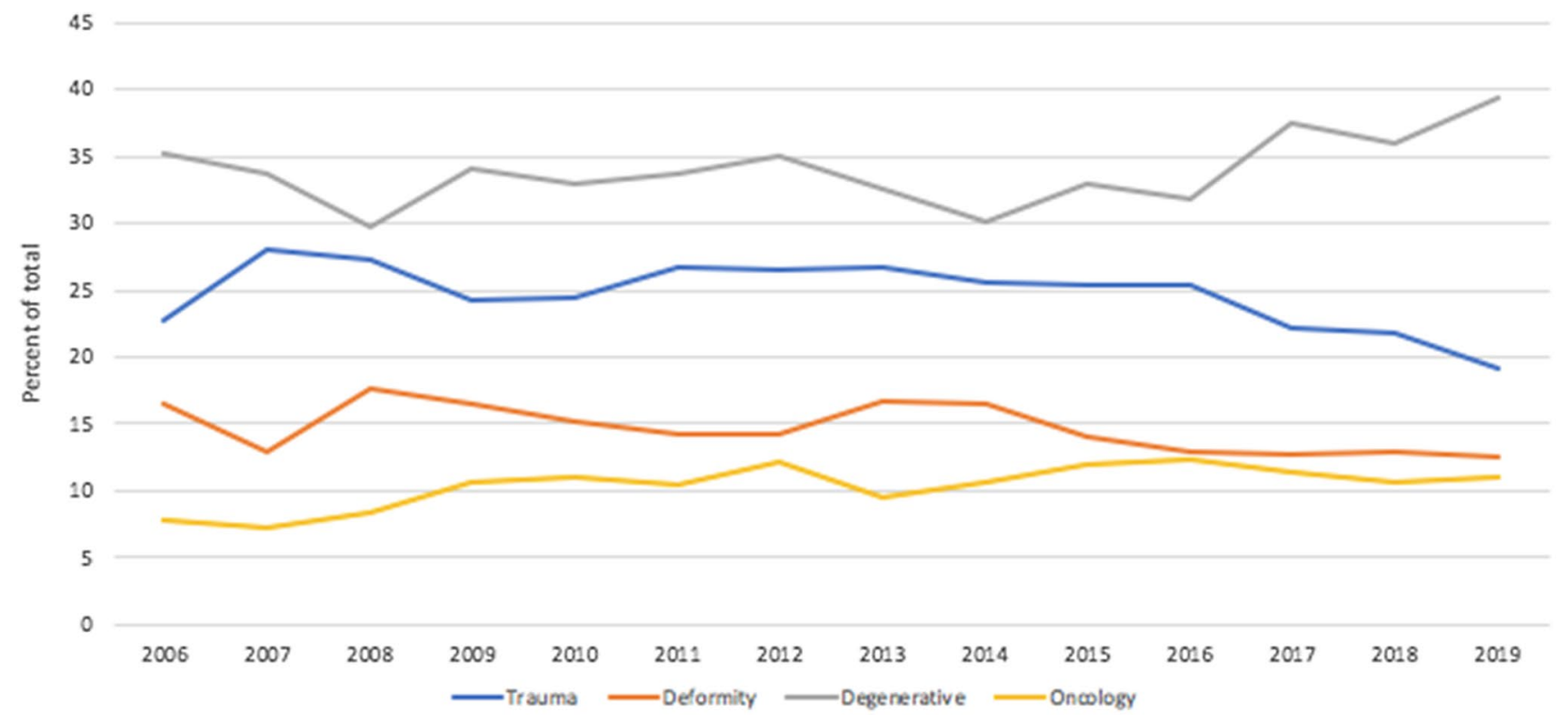

Fig. 1 Proportions of diagnostic category over time 
60

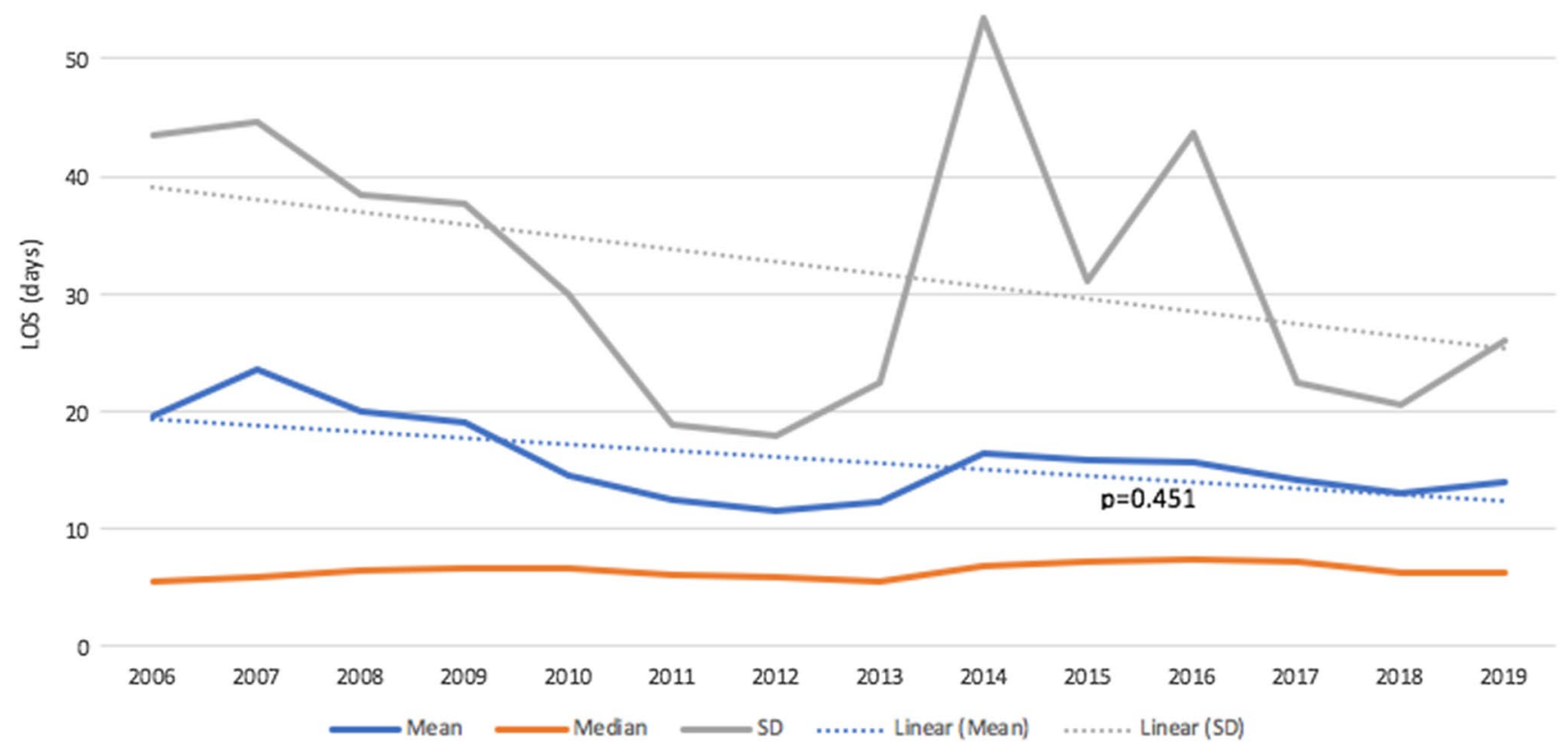

Fig. 2 Trends in LOS from 2006-2019

Table 2 Trends in length of stay (days) from 2006-2019

\begin{tabular}{llllll}
\hline Year & $\begin{array}{l}\text { Total } \\
\text { patients } \\
(\mathrm{N})\end{array}$ & Mean LOS & Median LOS & SD & IQR \\
\hline 2006 & 978 & 19.59 & 5.44 & 43.51 & 12.19 \\
2007 & 875 & 23.48 & 5.88 & 44.60 & 20.53 \\
2008 & 938 & 19.92 & 6.45 & 38.48 & 14.50 \\
2009 & 984 & 19.06 & 6.64 & 37.75 & 13.00 \\
2010 & 895 & 14.54 & 6.68 & 29.95 & 10.96 \\
2011 & 925 & 12.49 & 6.16 & 18.91 & 11.68 \\
2012 & 903 & 11.52 & 5.92 & 17.97 & 10.55 \\
2013 & 921 & 12.28 & 5.56 & 22.39 & 10.85 \\
2014 & 992 & 16.46 & 6.76 & 53.43 & 13.18 \\
2015 & 965 & 15.94 & 7.18 & 31.11 & 13.54 \\
2016 & 916 & 15.59 & 7.31 & 43.59 & 12.71 \\
2017 & 978 & 14.11 & 7.25 & 22.47 & 12.88 \\
2018 & 1105 & 12.99 & 6.19 & 20.56 & 11.15 \\
2019 & 1118 & 13.97 & 6.27 & 25.93 & 12.59 \\
\hline
\end{tabular}

not changed over time for patients treated for oncological disease.

Increasing age was significantly associated with increased number of adverse events (correlation 0.12, 95\% $\mathrm{CI}=0.07-0.17, p<0.001)$. Despite the significant increase in age of the study population, the number of patients having any adverse events has not significantly changed over time. On multivariate analysis, adjusted for age, each additional adverse event increased the LOS by 1 day $(p<0.001)$. This trend was observed in each diagnostic category (deformity, degenerative, oncology, trauma, and others). Concurrently, overall, surgical site infections (SSI) have significantly decreased over the study period from 7.9 to $1.4 \%$ $(p=<0.001)$. Similarly, SSI has decreased for deformity, degenerative, and oncology. However, SSI has not significantly changed for trauma.

\section{Discussion}

This study provides comprehensive and granular insights into the trends in and determinants of LOS in a high-volume quaternary spinal care center over the past 14 years. For the entire study period, mean LOS has not changed. This information along with the changing case mix is critical for current and future strategic planning and resource allocation. Prolonged LOS increases health care expenditure: thus, surgeons and providers are always seeking ways to improve cost-effectiveness while maintaining or improving outcomes in an era of rising healthcare costs.

Age has been reported as a key determinant of prolonged LOS in the literature $[1,4,7,10,23-25]$. This study demonstrated that elderly patients represent an increasing proportion of those receiving spine surgery. Spinal disease in the elderly most commonly includes degenerative disease such as lumbar spinal stenosis, cervical spondylosis, degenerative spondylolisthesis, and spinal 


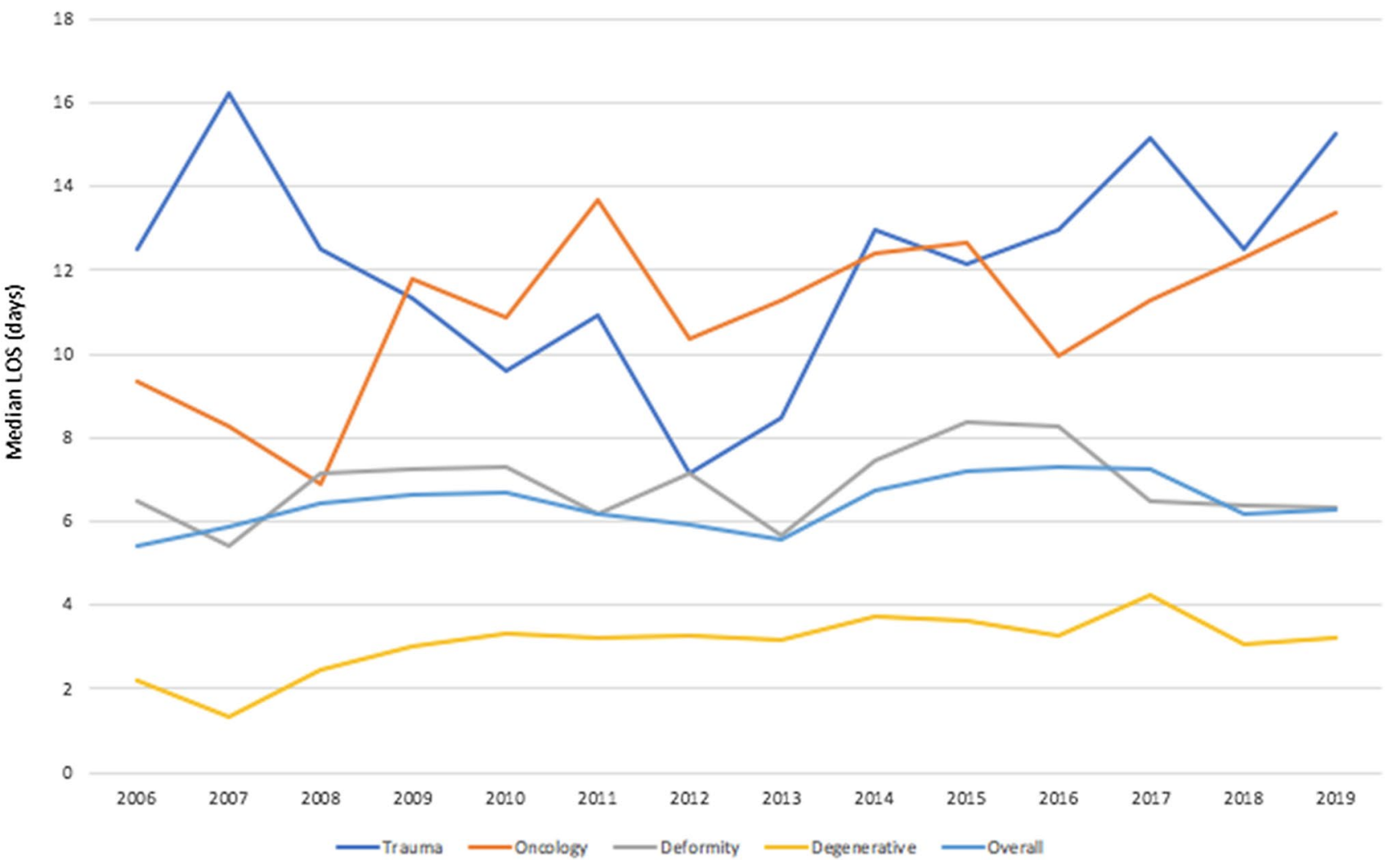

Fig. 3 Trends in median LOS per diagnostic category from 2006-2019

deformity [26, 27]. The elderly, who tend to be more frail and present with a greater burden of medical comorbidities may have difficulty with rehabilitation, increased risk of poor wound healing, and other complications [3, $7,28]$. The present study identified a significant correlation between increasing age and more adverse events. Surprisingly, adverse events have not increased over time despite increasing age of the treated population. Therefore, technological advances and hospital-based protocols aimed at reducing adverse events seem to have positively impacted patient outcomes [18]. For example, CT-based navigation was introduced in 2008; nasal decolonization in 2014; intrawound antibiotics in 2016; and silver-coated catheter in 2018.

A growing elderly population means more bed days are occupied per year as shown by the present study. Hospital administrators should focus on care pathways that target specific needs of the elderly population such as customized rehabilitation, nutrition optimization, and deconditioning prevention. Adogwa et al. reported on the co-management by spine surgeons and geriatricians of elderly patients undergoing lumbar fusion surgery with a Perioperative Optimization of Senior Health (POSH) program [29]. Their protocol reduced LOS by $30 \%$ through earlier and improved mobilization and reduction in postoperative complications.
In recent decades, the aging North American population has led to an increase in the number of spinal surgeries performed [30-35]. The growing burden on the healthcare system requires age-appropriate quality improvement initiatives. Minimizing LOS has long been seen as a key component of cost containment and service delivery [36]. For healthcare administrators, the objective would be to reduce LOS to improve hospital performance and efficiency of the care delivery process. This study has shown an increase in emergency surgeries for degenerative pathology over time. This may be the result of increasing demand for spinal surgeries, disproportionate to the limited resources available to provide them [37, 38]. Compromised access to surgeons whose waitlists for assessment and elective surgery are inundated with patients may lead to increases in patients with degenerative spinal pathology that would ideally be treated electively, presenting to emergency rooms in states of crisis.

To our knowledge, Kobayashi et al. report the only other large-scale study of trends in LOS in spinal surgery [4]. Their report shows a decrease in LOS in 10,829 patients over 10 years in Japan from 2005 to 2015, a similar time frame to the present study (2006-2019). In this Japanese study, the initial mean LOS was 25.9 days in 2005 with a median age in Japan of 43.0 years [39]. The LOS decreased to a mean of 19.6 days in 2015 with a median age in Japan 
of 46.4 years. This study does not present the trends in age in the population receiving spine surgery. In the present study, the initial LOS was 19.6 days in 2006 with a median age in Canada of 39.5 years [40]. The LOS was 14.0 days in 2019 with a median age of 40.5 years. First, LOS was defined as the number of calendar days from the operation to hospital discharge in the Japanese study, and LOS was defined by the number of calendar days from hospital admission to hospital discharge in this study. The longer LOS in the Japanese study may be explained by higher number of hospital beds per capita, which decreases the pressure to discharge. The decrease in LOS observed in the Japanese study is explained by the authors by a Japanese fixed payment public system, which is now a national medical policy. Secondly, the average LOS had more room for improvement in the Japanese study with a higher starting point (25.9 days vs 19.6 days).

When assessing trends in LOS, it is also necessary to assess trends in variability within the population as a whole, and within diagnostic subgroups. Declining or minimal variability may indicate little room for improvement, whereas greater variability may represent opportunity for improvement. In the present study, variability has been increasing in degenerative pathologies; conversely, it has remained stable in deformity, trauma, and oncology. This likely reflects the inherent heterogeneity of degenerative pathology which includes, for example, lumbar disc herniation, and cervical myelopathy. This phenomenon may also be explained in part by the increasing proportion of degenerative cases performed in an emergency setting. Emergent admission and treatment of degenerative conditions will add pre-operative hospital days while the patient obtains imaging or is waiting for urgent unscheduled surgical time. In contradistinction, elective patients are admitted on the day of surgery. The introduction of innovations that reduce LOS such as infection prevention protocols and intraoperative neuronavigation may have reduced the variability observed in LOS.

While the present study provides useful insight into trends in LOS over time, its findings should be interpreted within the context of the study design. Functional discharge status and readmissions were not assessed. Occasional transfer of patients to other facilities post-surgery was not considered and thus the LOS reported is not representative of time in hospital up until return to home. It is possible that an increased LOS could have a positive impact such as a lower readmission rate and improved functional status at discharge. Changes in institutional preoperative practices and procedures such as admission of patients from remote locations prior to surgery may have differentially influenced LOS over the study. However, in future studies, if LOS is operationally defining as the number of days from operation to discharge it could mitigate this potential source of confounding.

It is probable that length of stay was affected by delays in transfer to other hospitals or rehabilitation facilities due to external factors such as weather, limitations in transportation resources, or bed availability. Equally, some study patients likely continued receiving care at another hospital which is not accounted in the measured length of stay. However, we have no reason to believe that the presence or magnitude of such delays nor the duration of stay in community hospitals after discharge from our center would have changed over the study interval. Future studies should assess trends in adverse events and perform analysis on factors associated with increased LOS in each diagnostic category.

\section{Conclusion}

This is the first North American study to comprehensively analyze trends in LOS over time in spinal surgery. On aggregate, LOS has not changed from 2006-2019. Various factors that influence LOS appear to have balanced each other. It may also be explained by the changing epidemiology of both elective and emergency surgeries. These findings provide opportunities for intervention and improvement, targeted at the geriatric populations, to reduce length of hospitalization.

Acknowledgments This work would not have been possible without the dedication and hard work of the staff of the Vancouver Spine Research Group.

Funding This work was funded by the Bob and Trish Saunders Spine Research Fund through The VGH and UBC Hospital Foundation.

\section{Declarations}

Conflict of interest None of the authors has any potential conflict of interest.

\section{References}

1. Kobayashi K, Ando K, Kato F et al (2019) Predictors of prolonged length of stay after lumbar interbody fusion: a multicenter study. Glob Spine J 9:466-472. https://doi.org/10.1177/2192568218 800054

2. Inglis T, Banaszek D, Rivers CS et al (2020) In-hospital mortality for the elderly with acute traumatic spinal cord injury. J Neurotrauma 37:2332-2342. https://doi.org/10.1089/neu.2019.6912

3. Banaszek D, Inglis T, Marion TE et al (2020) Effect of frailty on outcome after traumatic spinal cord injury. J Neurotrauma 37:839-845. https://doi.org/10.1089/neu.2019.6581

4. Kobayashi K, Ando K, Kato F et al (2019) Trends of postoperative length of stay in spine surgery over 10 years in Japan based on a prospective multicenter database. Clin Neurol Neurosurg 177:97-100. https://doi.org/10.1016/j.clineuro.2018.12.020

5. van de Vijsel AR, Heijink R, Schipper M (2015) Has variation in length of stay in acute hospitals decreased? Analysing trends in the variation in LOS between and within Dutch hospitals. BMC Health Serv Res 15:438. https://doi.org/10.1186/ s12913-015-1087-6 
6. Shin JIB, Kim JS, Steinberger J et al (2018) Patient factors contributing to prolonged postoperative length of stay and increased rate of readmission after elective posterior cervical fusion. Clinical Spine Surg. https://doi.org/10.1097/BSD.0000000000000512

7. Basques BA, Varthi AG, Golinvaux NS et al (2014) Patient characteristics associated with increased postoperative length of stay and readmission after elective laminectomy for lumbar spinal stenosis. Spine (Phila Pa 1976) 39:833-840. https://doi.org/10.1097/ BRS.0000000000000276

8. Kim RB, Wilkerson C, Karsy M et al (2020) Prolonged length of stay and risk of unplanned 30-day readmission after elective spine surgery: propensity score-matched analysis of 33,840 patients. Spine 45:1260-1268. https://doi.org/10.1097/BRS.0000000000 003520

9. Lovecchio F, Steinhaus M, Elysee JC et al (2020) Factors associated with short length of stay after long fusions for adult spinal deformity: initial steps toward developing an enhanced recovery pathway. Glob Spine J. https://doi.org/10.1177/2192568220 941448

10. McGirt MJ, Parker SL, Chotai S et al (2017) Predictors of extended length of stay, discharge to inpatient rehab, and hospital readmission following elective lumbar spine surgery: introduction of the carolina-semmes grading scale. J Neurosurg Spine 27:382-390. https://doi.org/10.3171/2016.12.SPINE16928

11. Halpin RJ, Sugrue PA, Gould RW et al (2010) Standardizing care for high-risk patients in spine surgery: the Northwestern high-risk spine protocol. Spine (Phila Pa 1976) 35:2232-2238. https://doi. org/10.1097/BRS.0b013e3181e8abb0

12. Fisher CG, Vaccaro AR, Whang PG et al (2013) Evidencebased recommendations for spine surgery. Spine (Phila Pa 1976) 38:E30-37. https://doi.org/10.1097/BRS.0b013e318275cdd8

13. Sugrue PA, Halpin RJ, Koski TR (2013) Treatment algorithms and protocol practice in high-risk spine surgery. Neurosurg Clin N Am 24:219-230. https://doi.org/10.1016/j.nec.2012.12.012

14. Sethi R, Buchlak QD, Yanamadala V et al (2017) A systematic multidisciplinary initiative for reducing the risk of complications in adult scoliosis surgery. J Neurosurg Spine 26:744-750. https:// doi.org/10.3171/2016.11.SPINE16537

15. Buchlak QD, Yanamadala V, Leveque J-C, Sethi R (2016) Complication avoidance with pre-operative screening: insights from the Seattle spine team. Curr Rev Musculoskelet Med 9:316-326. https://doi.org/10.1007/s12178-016-9351-x

16. Dietz N, Sharma M, Adams $S$ et al (2019) Enhanced recovery after surgery (ERAS) for spine surgery: a systematic review. World Neurosurg 130:415-426. https://doi.org/10.1016/j.wneu.2019.06. 181

17. Elsarrag M, Soldozy S, Patel P et al (2019) Enhanced recovery after spine surgery: a systematic review. Neurosurg Focus 46:E3. https://doi.org/10.3171/2019.1.FOCUS18700

18. Dea N, Fisher CG, Batke J et al (2016) Economic evaluation comparing intraoperative cone beam CT-based navigation and conventional fluoroscopy for the placement of spinal pedicle screws: a patient-level data cost-effectiveness analysis. Spine J 16:23-31. https://doi.org/10.1016/j.spinee.2015.09.062

19. Ehlers AP, Khor S, Shonnard $N$ et al (2016) Intra-wound antibiotics and infection in spine fusion surgery: a report from Washington State's SCOAP-CERTAIN collaborative. Surg Infect (Larchmt) 17:179-186. https://doi.org/10.1089/sur.2015.146

20. Gometz A, Maislen D, Youtz C et al (2018) The effectiveness of prehabilitation (Prehab) in both functional and economic outcomes following spinal surgery: a systematic review. Cureus 10:e2675. https://doi.org/10.7759/cureus.2675

21. Lindbäck Y, Tropp H, Enthoven P et al (2018) PREPARE: presurgery physiotherapy for patients with degenerative lumbar spine disorder: a randomized controlled trial. Spine J 18:1347-1355. https://doi.org/10.1016/j.spinee.2017.12.009
22. Services $\mathrm{M}$ of $\mathrm{C}$ Population Estimates-Province of British Columbia. https://www2.gov.bc.ca/gov/content/data/statistics/ people-population-community/population/population-estimates. Accessed 22 Feb 2021

23. Zheng F, Cammisa FPJ, Sandhu HS et al (2002) Factors predicting hospital stay, operative time, blood loss, and transfusion in patients undergoing revision posterior lumbar spine decompression, fusion, and segmental instrumentation. Spine 27:818-824

24. Gruskay JA, Fu M, Bohl DD et al (2015) Factors affecting length of stay after elective posterior lumbar spine surgery: a multivariate analysis. Spine J 15:1188-1195. https://doi.org/10. 1016/j.spinee.2013.10.022

25. Dial BL, Esposito VR, Danilkowicz R et al (2020) Factors associated with extended length of stay and 90-day readmission rates following ACDF. Glob Spine J 10:252-260. https:// doi.org/10.1177/2192568219843111

26. O'Lynnger TM, Zuckerman SL, Morone PJ et al (2015) Trends for spine surgery for the elderly: implications for access to healthcare in North America. Neurosurgery 77:S136-S141. https://doi.org/10.1227/NEU.0000000000000945

27. Deyo RA, Cherkin DC, Loeser JD et al (1992) Morbidity and mortality in association with operations on the lumbar spine. The influence of age, diagnosis, and procedure. J Bone Joint Surg Am 74:536-543

28. Raffo CS, Lauerman WC (2006) Predicting morbidity and mortality of lumbar spine arthrodesis in patients in their ninth decade. Spine (Phila Pa 1976) 31:99-103. https://doi.org/10.1097/ 01.brs.0000192678.25586.e5

29. Adogwa O, Elsamadicy AA, Vuong VD et al (2017) Geriatric comanagement reduces perioperative complications and shortens duration of hospital stay after lumbar spine surgery: a prospective single-institution experience. J Neurosurg Spine 27:670-675. https://doi.org/10.3171/2017.5.SPINE17199

30. Johnson WC, Seifi A (2018) Trends of the neurosurgical economy in the United States. J Clin Neurosci 53:20-26. https://doi. org/10.1016/j.jocn.2018.04.041

31. Deyo RA (2010) Trends, major medical complications, and charges associated with surgery for lumbar spinal stenosis in older adults. JAMA 303:1259. https://doi.org/10.1001/jama. 2010.338

32. Marquez-Lara A, Nandyala SV, Fineberg SJ, Singh K (2014) Current trends in demographics, practice, and in-hospital outcomes in cervical spine surgery: a national database analysis between 2002 and 2011. Spine (Phila Pa 1976) 39:476-481. https://doi.org/10. 1097/BRS.0000000000000165

33. Patil PG, Turner DA, Pietrobon R (2005) National trends in surgical procedures for degenerative cervical spine disease: 1990 2000. Neurosurgery 57:753-758

34. Zeidman SM, Ducker TB, Raycroft J (1997) Trends and complications in cervical spine surgery: 1989-1993. J Spinal Disord $10: 523-526$

35. O'Lynnger TM, Zuckerman SL, Morone PJ et al (2015) Trends for spine surgery for the elderly: implications for access to healthcare in North America. Neurosurgery 77(Suppl 4):S136-141. https:// doi.org/10.1227/NEU.0000000000000945

36. Puffer RC, Planchard R, Mallory GW, Clarke MJ (2016) Patientspecific factors affecting hospital costs in lumbar spine surgery. $\mathrm{J}$ Neurosurg Spine 24:1-6. https://doi.org/10.3171/2015.3.SPINE 141233

37. Reisener M-J, Pumberger M, Shue J et al (2020) Trends in lumbar spinal fusion-a literature review. J Spine Surg 6:752-761. https:// doi.org/10.21037/jss-20-492

38. Xu Y, Yen D, Whitehead M et al (2019) Use of instrumented lumbar spinal surgery for degenerative conditions: trends and costs over time in Ontario, Canada. Can J Surg 62:393-401. https://doi. org/10.1503/cjs.017016 
39. Japan Population (2021) Worldometer. https://www.worldometers. info/world-population/japan-population/. Accessed 13 Apr 2021

40. Government of Canada SC (2007) Statistics Canada: age and sex highlight tables, 2006 Census. https://www12.statcan.gc.ca/ census-recensement/2006/dp-pd/hlt/97-551/index.cfm?Lang=E. Accessed 13 Apr 2021
Publisher's Note Springer Nature remains neutral with regard to jurisdictional claims in published maps and institutional affiliations. 